\title{
Evaluation of Antioxidants Status at Diagnosis in Childhood Acute Lymphoblastic Leukemia
}

\author{
Elhamy Rifky Abdelkhalek ${ }^{1}$, Yosry El-Sayed Abo-Elmagd ${ }^{2}$, Adel Sherif Ahmed ${ }^{1}$, Mohamed Mostafa Arafa*1 \\ Departments of ${ }^{1}$ Pediatrics, ${ }^{2}$ Biochemistry, Faculty of Medicine, Zagazig University, Egypt. \\ *Corresponding Author: Mohamed M. Arafa, Mobile: (+20)01140006686, Email: m.arafa86@gmail.com
}

\begin{abstract}
Background: Acute lymphoblastic leukemia (ALL) is a neoplastic disease of immature lymphocytes or lymphocyte progenitor cells either the B- or T-cell lineage. It is the most common malignancy diagnosed in patients younger than 15 years, and account for approximately $25-30 \%$ of adult acute leukemia.

Objective: To evaluate some antioxidants as zinc, selenium, vitamin A (retinol) and vitamin E (tocopherol) status at diagnosis of childhood acute lymphoblastic leukemia and to compare it to that of a control population.

Patients and methods: This case-control study was carried out at Oncology Unit of Pediatrics Department and Medical Biochemistry Department, Faculty of Medicine, Zagazig University Hospitals. This study consisted of two groups: Patients' group (30 children patients with newly diagnosed ALL). As regard control group, it included 30 healthy children who were matched well with patients' age and sex. Fasting levels of serum zinc, selenium, retinol and tocopherol were measured.

Results: There was a high statistically significant decrease of vitamin E, selenium and zinc among all cases than their controls. While, there was a significant increase of vitamin A among all cases. Zinc level was statistically significantly increased with increased risk of disease, while no statistically significant relation between disease risk and other levels of serum antioxidant. Conclusion: Some antioxidants as zinc, selenium and vitamin E (tocopherol) status at diagnosis of childhood acute lymphoblastic leukemia are lower compared to that of a control population.
\end{abstract}

Key words: Selenium, Retinol, Tocopherol, Antioxidants, Acute lymphoblastic leukemia.

\section{INTRODUCTION}

Every year more than 175300 children are diagnosed with malignancy in the world. Of this total, 80 percent of children with malignancy, live in developing countries including Egypt and contribute significantly to the estimated 96400 childhood cancer deaths annually. Thus childhood malignancies are considered an important global public health problem [1]. Acute lymphoblastic Leukemia (ALL) is the most common cancer found in children under 15 years of age and it accounts for more than 50\% of the hematopoietic malignancies in this age group. ALL is a disorder caused by an abnormal expression of genes, which is usually a result of chromosomal translocation. The disease can be originated from lymphoid cells of different lineages giving rise to $\mathrm{B}$ or $\mathrm{T}$ cell Leukemia or sometimes mixed-lineage leukemia ${ }^{[2]}$.

Selenium (Se) is a trace element that share in the antioxidant system by saving the cells from reactive oxygen species. The possibility that increased supplementation of selenium and vitamin E can protect against the development of cancer has generated great interest. Low serum selenium levels appear in children with malignancies ${ }^{[3]}$.

Vitamin A (Retinol) and Vitamin E (Tocopherol) have been studied in relation to cancer chemotherapy and toxicity reduction. In a study, supplementation of tocopherol has shown reduced hematological complications and need for transfusion support in the first 2 months of treatment ${ }^{[4]}$.
Zinc also has been shown to have immunomodulatory effects both directly by acting on the immune system and indirectly by acting on the respiratory, gastrointestinal and epithelial linings. Several studies have shown benefits of long-term zinc supplementation on the incidence, severity, and duration of diarrhea and respiratory infections. Prolonged zinc supplementation also improves cellmediated immunity in severely malnourished children [5]. Zinc may also reduce the duration and severity of febrile neutropenia and associated complications ${ }^{[6]}$.

Patients who suffer from malnutrition have been shown to tolerate treatment with chemotherapeutic drugs poorly. Most studies focusing on nutrition and outcome in pediatric cancer have focused on the clinical nutritional status of the patient and few have looked at the serum micronutrient levels at diagnosis [7].

Cancer patients have been noted to have low antioxidant levels even at initial diagnosis ${ }^{[8]}$. Oxidant damage with free radicals leads not only to cancers but also is implicated in chemotherapy-related adverse effects ${ }^{[9]}$. Children with normal dietary intake of antioxidants while on treatment for cancers have fewer complications and better quality of life ${ }^{[7]}$. Some argue that since many chemotherapeutic drugs act by producing reactive oxygen species, antioxidants may be counter-productive in that they may decrease the efficacy of chemotherapy [10]. However other studies find that it is helpful in reducing the side effects of chemotherapy. Patients on antioxidants tolerate 
chemotherapy better and thus require lesser dose reductions or suffer lesser breaks in treatment schedule. This, in turn, improves the response to treatment and survival ${ }^{[11]}$.

The aim of this work was to evaluate some antioxidants as zinc, selenium, vitamin A (retinol) and vitamin $\mathrm{E}$ (tocopherol) status at diagnosis of childhood acute lymphoblastic leukemia and to compare it to that of a control population.

\section{PATIENT AND METHODS}

This case-control study was carried out at Oncology Unit of Pediatrics Department and Medical Biochemistry Department, Faculty of Medicine, Zagazig University Hospitals during the period from January 2017 to January 2018. This study consisted of two groups: patients' group (30 children patients with newly diagnosed ALL) their ages ranged from 5 years to 15 years old with a mean age of 7.8 years. The males constituted $63.3 \%$ of patients \& the females were $36.7 \%$. As regards control group, it included 30 subjects who were age- and sex-matched with patients' group.

Inclusion criteria: Newly diagnosed patients of ALL aged $<18$ years at diagnosis were included in the study. Diagnosis of ALL was confirmed by morphological examination of bone marrow along with immuno-phenotyping wherever possible.

Exclusion criteria: Patients above 18 years of age. Patients already on antioxidant supplementation at the time of presentation. Patient on multivitamin supplementation in the past 4 weeks. Patients with abnormal growth parameters (height or weight $<3$ rd and $>$ 97th centile as growth charts). Patients with apparent antioxidant deficiency (Zinc), Patients with malabsorption syndrome.

\section{Ethical Approval:}

Written Informed consent was taken from the patients' parents to participate in the study. The permission for the study was given to the Pediatrics Departments of Zagazig University Hospitals after the permission of the Institutional Review Board (IRB). The research was carried out in compliance with the Code of Ethics of the World Medical Association (Declaration of Helsinki) for studies involving humans.

All included children were subjected to complete history taking, clinical examination, laboratory investigations including $\mathrm{CBC}$, blood smear examination, fasting levels of serum zinc, selenium, vit $\mathrm{E}$ and A. Anthropometric measurements: including weight (in $\mathrm{Kg}$ ) and length (in $\mathrm{cm}$ ) according to the world health organization recommendation. Specimens were centrifuged immediately after collection to obtain serum. The serum of each patient was kept frozen at -
$20 \mathrm{C}^{\circ}$. All samples were processed altogether after completion of collection.

\section{Methods:}

Serum zinc, selenium, vitamin E and vitamin A were measured at diagnosis in cases and in controls. Samples were obtained after overnight fasting in metal-free vials. The vials were kept upright in room temperature for $1 \mathrm{~h}$ following which, they were centrifuged at 1,300 rpm for 10 minutes. Separated serum was collected in metal-free vials with careful elimination of external contamination and stored at $-20{ }^{\circ} \mathrm{C}$ till analysis. Serum selenium, serum vitamin $\mathrm{E}$ and serum vitamin A levels were determined by using enzyme-linked immunosorbent assay (ELISA) technique. The kit was provided from SunRed Company, China. Serum Zinc level was determined by using colorimetric method with 5-Brom-PAPS. The kit was provided from Spectrum Diagnostics Company, China.

\section{Statistical Analysis}

Data were analyzed using IBM SPSS 23.0 for windows (SPSS Inc., Chicago, IL, USA) and NCSS 11 for windows (NCSS LCC., Kaysville, UT, USA). Quantitative data were expressed as mean \pm standard deviation (SD). Qualitative data were expressed as frequency and percentage. Independent sample t-test or Mann Whitney U test were used when comparing two means of not normally distributed data. Receiver operating characteristic (ROC) curve analysis was used to identify optimal cut-off values.

\section{RESULTS}

There was no statistical significant difference between ALL cases and controls regarding demographic data, age, sex, weight, height and BMI (Table 1). This study showed that $76.7 \%$ of studied patients had therapy delay and the commonest cause was thrombocytopenia (56.5\%) then leukopenia $(47.8 \%)$. There was $86.7 \%$ of studied patients presented with fever, $83.3 \%$ and $80 \%$ had hepatomegaly and splenomegaly respectively, $70 \%$ had pallor and $63.3 \%$ with lymphadenopathy (Table 2).

This study showed high statistically significant decrease of vitamin E, selenium and zinc among ALL cases than in their controls, while there was a significant increase of vitamin A among ALL cases (Table 3). There was no statistically significant difference among both types of ALL regarding levels of serum antioxidant (Table 4).

This study showed that zinc level was statistically significantly increased with increased risk of disease, while no statistically significant relation between disease risk and other levels of serum antioxidant (Table 5). 
Table (1): Demographic characters of both studied groups

\begin{tabular}{|c|c|c|c|c|}
\hline Variables & $\begin{array}{l}\text { ALL cases } \\
\text { Mean } \pm \text { SD }\end{array}$ & $\begin{array}{c}\text { Controls } \\
\text { Mean } \pm \text { SD }\end{array}$ & $\begin{array}{c}\text { t-test } \\
\text { MW }^{\#} \text { test }\end{array}$ & P-value \\
\hline $\begin{array}{c}\text { Age (years) } \\
\text { Range }\end{array}$ & $\begin{array}{c}7.8 \pm 3.58 \\
5-15 \\
\end{array}$ & $\begin{array}{c}9.2 \pm 3.53 \\
4-15 \\
\end{array}$ & $1.34^{\#}$ & $\begin{array}{c}0.145 \\
\text { NS } \\
\end{array}$ \\
\hline $\begin{array}{c}\text { Weight (kg) } \\
\text { Range }\end{array}$ & $\begin{array}{c}20.9 \pm 6.87 \\
10-38 \\
\end{array}$ & $\begin{array}{c}21.5 \pm 6.35 \\
12-35 \\
\end{array}$ & 0.37 & $\begin{array}{c}0.71 \\
\text { NS } \\
\end{array}$ \\
\hline $\begin{array}{c}\text { Height }(\mathrm{cm}) \\
\text { Range }\end{array}$ & $\begin{array}{c}112.9 \pm 19.1 \\
81-155\end{array}$ & $\begin{array}{c}114.2 \pm 17.6 \\
92-150\end{array}$ & 0.28 & $\begin{array}{c}0.79 \\
\text { NS }\end{array}$ \\
\hline $\begin{array}{c}\text { BMI }\left(\mathrm{kg} / \mathrm{m}^{2}\right) \\
\text { Range }\end{array}$ & $\begin{array}{c}16.6 \pm 1.92 \\
12.5-22.2\end{array}$ & $\begin{array}{c}16.99 \pm 2.01 \\
13-20\end{array}$ & 0.89 & $\begin{array}{c}0.39 \\
\text { NS }\end{array}$ \\
\hline & $\mathbf{N}(\%)$ & $\mathbf{N}(\%)$ & $\mathrm{X}^{2}$ & P-value \\
\hline $\begin{array}{c}\text { Male } \\
\text { Female }\end{array}$ & $\begin{array}{l}19(63.3 \%) \\
11(36.7 \%)\end{array}$ & $\begin{array}{l}18(60 \%) \\
12(40 \%)\end{array}$ & 0.071 & $\begin{array}{c}0.79 \\
\text { NS }\end{array}$ \\
\hline
\end{tabular}

Table (2): Events during induction assessed among the studied ALL cases

\begin{tabular}{|l|c|c|}
\hline \multirow{2}{*}{ Variables } & \multicolumn{2}{|c|}{ Studied group $(\mathbf{N}=\mathbf{3 0})$} \\
\cline { 2 - 3 } & $\mathbf{N}$ & $\mathbf{1}$ \\
\hline Therapy delay & & $\mathbf{7 6 . 7}$ \\
Yes & $\mathbf{2 3}$ & $\mathbf{2 3 . 3}$ \\
\hline No & $\mathbf{7}$ & \\
\hline Cause of delay: & $\mathbf{n}=\mathbf{2 3})$ & $\mathbf{5 6 . 5}$ \\
Thrombocytopenia & $\mathbf{1 3}$ & $\mathbf{2 1 . 7}$ \\
Anemia & $\mathbf{5}$ & $\mathbf{4 7 . 8}$ \\
Leukopenia & $\mathbf{1 1}$ & $\mathbf{1 3}$ \\
Leukocytosis & $\mathbf{3}$ & $\mathbf{8 6 . 7}$ \\
\hline Fever & $\mathbf{2 6}$ & $\mathbf{7 0}$ \\
\hline Pallor & $\mathbf{2 1}$ & $\mathbf{5 0}$ \\
\hline Bleeding & $\mathbf{1 5}$ & $\mathbf{8 3 . 3}$ \\
\hline Hepatomegaly & $\mathbf{2 5}$ & $\mathbf{8 0}$ \\
\hline Splenomegaly & $\mathbf{2 4}$ & \\
\hline
\end{tabular}

Table (3): Difference in anti-oxidant level among both studied groups

\begin{tabular}{|c|c|c|c|}
\hline Variables & $\begin{array}{c}\text { ALL cases } \\
\text { Mean } \pm \text { SD }\end{array}$ & $\begin{array}{c}\text { controls } \\
\text { Mean } \pm \text { SD }\end{array}$ & P-value \\
\hline Vitamin E (mg) & $\mathbf{0 . 8 5} \pm \mathbf{0 . 2}$ & $\mathbf{1 . 8 5} \pm 0.1$ & $<0.001$ HS \\
\hline Selenium (ug/dL) & $\mathbf{1 3 . 3} \pm 3.9$ & $\mathbf{2 4 . 4} \pm 4.5$ & $<0.001$ HS \\
\hline Vitamin A (mcg) & $\mathbf{6 7 . 3} \pm 13.6$ & $\mathbf{4 1 . 1} \pm 10.01$ & $<0.001$ HS \\
\hline Zinc (ug/dL) & $\mathbf{6 6 . 5} \pm 22.4$ & $\mathbf{1 1 8 . 4} \pm 10.2$ & $<0.001$ HS \\
\hline
\end{tabular}

HS: P-value $<0.001$ is high significant

Table (4): Relation between phenotyping of disease and antioxidant level of ALL cases

\begin{tabular}{|c|c|c|c|c|c|c|}
\hline & & $\mathbf{N}$ & Median & Mean & S.D & P-value \\
\hline \multirow{2}{*}{ Vit. E } & C-ALL & 21 & 0.81 & 0.91 & 0.16 & \multirow{2}{*}{$\begin{array}{c}0.397 \\
\text { NS }\end{array}$} \\
\hline & T-ALL & 9 & 0.68 & 0.71 & 0.22 & \\
\hline \multirow{2}{*}{ Selenium } & C-ALL & 21 & 12.7 & 12.5 & 2.34 & \multirow{2}{*}{$\begin{array}{c}0.563 \\
\text { NS }\end{array}$} \\
\hline & T-ALL & 9 & 15 & 15.2 & 3.45 & \\
\hline \multirow{2}{*}{ Vit. A } & C-ALL & 21 & 68 & 68.3 & 3.86 & \multirow{2}{*}{$\begin{array}{c}0.528 \\
\text { NS }\end{array}$} \\
\hline & T-ALL & 9 & 68 & 64.8 & 13.5 & \\
\hline \multirow{2}{*}{ Zinc } & C-ALL & 21 & 72 & 63.8 & 4.9 & \multirow{2}{*}{$\begin{array}{c}0.533 \\
\text { NS }\end{array}$} \\
\hline & T-ALL & 9 & 71.4 & 72.8 & 14.2 & \\
\hline
\end{tabular}

NS: P-value $>0.05$ is not significant $\quad$ MW: mann-whitney test of non-parametric data 
Table (5): Relation between risk of disease and antioxidant level of ALL cases

\begin{tabular}{|c|c|c|c|c|c|c|}
\hline & & $\mathbf{N}$ & Median & Mean & S.D & $P$ value \\
\hline \multirow[t]{3}{*}{ Vit. E } & Low & 9 & 0.82 & 0.81 & 0.096 & \multirow{3}{*}{$0.194 \mathrm{NS}$} \\
\hline & Standard & 13 & 0.7 & 0.86 & 0.05 & \\
\hline & High & 8 & 0.89 & 0.85 & 0.29 & \\
\hline \multirow[t]{3}{*}{ Selenium } & Low & 9 & 13.4 & 12.7 & 2.93 & \multirow{3}{*}{$0.141 \mathrm{NS}$} \\
\hline & Standard & 13 & 9.7 & 12.1 & 2.3 & \\
\hline & High & 8 & 16 & 15.8 & 3.99 & \\
\hline \multirow[t]{3}{*}{ Vit. A } & Low & 9 & 73.2 & 75.2 & 14.9 & \multirow{3}{*}{$0.83 \mathrm{NS}$} \\
\hline & Standard & 13 & 67.7 & 64.4 & 10.5 & \\
\hline & High & 8 & 64.9 & 62.98 & 14.4 & \\
\hline \multirow[t]{3}{*}{ Zinc } & Low & 9 & 63.7 & 65.7 & 16.7 & \multirow{3}{*}{$0.01 \mathrm{~S}$} \\
\hline & Standard & 13 & 71.4 & 61.8 & 8.1 & \\
\hline & High & 8 & 73.9 & 74.9 & 16.8 & \\
\hline
\end{tabular}

\section{DISCUSSION}

In the present study, the patients group included 30 patients their age ranged from 5 years to 15 years old with a mean age of 7.8 years. The males constituted $63.3 \%$ of patients \& the females were $36.7 \%$. The control group included 30 subjects who were matching well with patients as regard age and sex. This comes in agreement with Khaled ${ }^{[12]}$, Alleban et $\boldsymbol{a l} .{ }^{[13]}$ and Willman et al. ${ }^{[14]}$ who reported male predominance in ALL patients. Ten Hacken and Burger ${ }^{[15]}$ found that childhood leukemia occurred slightly more frequently in boys than girls. The reason which may be assisting the male to be infected with leukemia could be due to presence of the sex responsive gene near gene $\mathrm{BCR}-\mathrm{ABL}$, which is located on a Philadelphia chromosome ${ }^{[3]}$. A study conducted in Baghdad Teaching Hospital showed that more male dominance than female may be due to the nature of their hormones ${ }^{[16]}$.

In the present study, no statistically significant difference between ALL cases and controls regarding demographic data, age, sex, weight, height, and BMI. This comes in agreement with Radhakrishnan et al. ${ }^{[3]}$ who found that there was no significant difference between ALL cases and controls regarding demographic data.

In the present study, in general, the index study population was nutritionally comparable to the control population as observed by similar anthropometric measurements. More detailed anthropometry and dietary information was beyond the scope of the present study as this was undertaken as a pilot project. Children with ALL present usually within 2-3 weeks of onset of symptoms and are hence rarely malnourished at diagnosis as a result of the illness. After the onset of chemotherapy, adverse effects such as mucositis, nausea and vomiting set in and dietary intake reduces ${ }^{[7]}$. Hence, we compared only serum levels in patients taken at initial diagnosis, with that of normal controls.

In the current study, the main clinical presentations among the newly diagnosed ALL patients were fever in $86.7 \%$ of patients, followed by liver enlargement (83.3\%), splenomegaly (80\%), pallor $(70 \%)$ and lymphadenopathy $(63.3 \%)$. The least clinical datum was bleeding (50\%). There were no any CNS manifestations. This incidence is comparable to that reported by an Egyptian study done by Ahmed and Hassab ${ }^{[17]}$ who detected lymphadenopathy in $73.3 \%$ of their patients and CNS infiltration in only $6.7 \%$ of patients.

In our study, there was $76.7 \%$ of studied patients had therapy delay and the commonest cause was thrombocytopenia (56.5\%) then leukopenia (47.8\%).

In the current study, there was a high statistically significant decrease of vitamin $\mathrm{E}$, selenium and zinc among ALL cases more than in controls, while there was a significant increase of vitamin A among ALL cases. In agreement with our study, several studies have demonstrated lower levels of serum selenium, zinc and vitamin $\mathrm{E}$ in patients with malignancies compared to controls ${ }^{[18,}{ }^{19]}$. Even studies evaluating their selenium levels have found lower levels in patients with cancers compared to normal controls ${ }^{[20]}$. It has often been suggested that pre-treatment value of zinc and selenium is inversely proportional to the peripheral blast cell count, indicating that it is a measure of tumor burden ${ }^{[9]}$. A similar result was shown by Singh et al. ${ }^{[21]}$ who found significantly decreased serum vitamin $\mathrm{E}$ levels in chronic myeloid leukemia patients before starting treatment. These findings confirm that vitamin $\mathrm{E}$ is an important antioxidant that is altered in leukemia. As an antioxidant, vitamin E may inhibit cancer formation by scavenging reactive oxygen or nitrogen species and could be considered the major membrane-bound 
antioxidant employed by the cell. Also, experimental data support the presence of slight zinc deficiency in malignancies ${ }^{[22]}$. Zinc deficiency may cause disturbances in oxidation, mitochondrial function, DNA repair, and cancer induction and progression. Impaired zinc metabolism in the pathogenesis of leukemia was identified in 1949. Zinc seems to improve the overall ability of the patients in resisting the toxic side effects of chemotherapy ${ }^{[23]}$. Also, in disagreement with our study, Akhgarjand et al. ${ }^{[25]}$ found that the concentration of serum zinc was the same in the patients and healthy individuals. Results of another study showed that serum zinc concentrations were similar in patients with ALL and healthy individuals ${ }^{[3]}$.

Poor nutritional status, which is shown through body composition, is an important concern for the patients with cancer. Nutritional status is associated with decreased tolerance of chemotherapy, increased susceptibility to infection, and poor clinical results. Nutritional problems are different based on the type of cancer and its treatment and may be a concern at the time of diagnosis ${ }^{[24]}$.

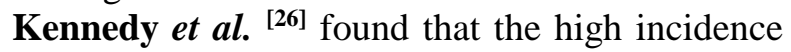
of plasma retinol deficiency at diagnosis is likely related to the low levels of retinol binding protein (RBP) concentration at diagnosis. Fifty to eighty percent of vitamin A in the body is stored in the liver for use during periods of decreased intake. In order for the stored vitamin A to be mobilized from the liver it must be de-esterified and bound to retinol binding protein (RBP). Children with cancer, including ALL, have lower concentrations of RBP at diagnosis as compared to healthy controls. In disagreement with our study, Radhakrishnan et al. ${ }^{[3]}$ observed similar levels of serum zinc, selenium, vitamins $\mathrm{A}$ and $\mathrm{E}$ in both groups of patients and control. There were minor differences in the levels between the groups, but they were not significant statistically. Few studies that have focused on patients with ALL alone have reported similar values of zinc and alpha tocopherol between patients and normal controls at diagnosis ${ }^{[27,28]}$.

Antioxidants, although not used as part of treatment are taken by a majority of patients, often following medical advice. Antioxidants also show promise in cancer therapy by reducing the painful side effects of treatment ${ }^{[29]}$. Almost all anticancer drugs work by affecting the DNA synthesis. The potential effect of antioxidants in modifying treatment efficacy would depend on the type of drug. Supplementation of vitamins has been found to be protective against mucositis and other radiation side effects. Zinc supplementation has also shown improvement in mucositis and dermatitis following radiation therapy in patients with head and neck cancers ${ }^{[30]}$.

\section{CONCLUSION}

We concluded that some antioxidants as zinc, selenium and vitamin E (tocopherol) status at diagnosis of childhood acute lymphoblastic leukemia were lower compared to that of a control population.

Financial support and sponsorship: Nil. Conflict of interest: Nil.

\section{REFERENCES}

1. Ibrahim A, Khaled H, Mikhail $\mathbf{N}$ et al. (2014). Cancer Incidence in Egypt: Results of the National Population-Based Cancer Registry Program. J Cancer Epidemiol., 18: 1-5.

2. El-Sabagh M, Ramadan K, El-slam I et al. (2011): Antioxidants Status in Acute Lymphoblastic Leukemia Patients. American J Med Sci., 1 (1): 1-6.

3. Radhakrishnan N, Dinand V, Rao S et al. (2013): Antioxidant levels at diagnosis in childhood acute lymphoblastic leukemia. Indian J Pediatr., 80: 292-6.

4. Al-Tonbary Y, Al-Haggar M, El-Ashry $\mathrm{R}$ et al. (2009): Vitamin e and N-acetylcysteine as antioxidant adjuvant therapy in children with acute lymphoblastic leukemia. Advances in Hematology, 9: 1-6.

5. Yakoob M, Theodoratou E, Jabeen A et al. (2011): Preventive zinc supplementation in developing countries: impact on mortality and morbidity due to diarrhea, pneumonia and malaria. BMC Public Health, 11 (3): 23-26.

6. Kulkarni K, Marwaha R, Trehan A et al. (2009): Survival outcome in childhood ALL: experience from a tertiary care center in North India. Pediatr Blood Canc., 53: $168-73$.

7. Kennedy D, Tucker K, Ladas E et al. (2004): Low antioxidant vitamin intakes are associated with increases in adverse effects of chemotherapy in children with acute lymphoblastic leukemia. Am J Clin Nutr., 79: 1029-36.

8. Ladas E, Jacobson J, Kennedy D et al. (2004): Antioxidants and cancer therapy: a systematic review. J Clin Oncol., 22(3): 517-528.

9. Zuo X, Chen J, Zhou $X$ et al. (2006): Levels of selenium, zinc, copper and antioxidant enzyme activity in patients with leukemia. Biol Trace Elem Res., 114: 41-53.

10. Gabriella M (2005): Use of Antioxidants during Chemotherapy and Radiotherapy Should Be Avoided. A Cancer J Clin., 55 (5): 319-21.

11. Kenneth A (2004): Chemotherapy-Associated Oxidative Stress: Impact on Chemotherapeutic Effectiveness. Integr Cancer Ther., 3 (4): 294-300.

12. Khaled S (2013): Abo and Rhesus Blood Groups. Possible Entities in the World Health Organization Classification Acutenon - Lymphoblastic Leukemia. Journal of Research in Medical Sciences, 11 (4): 239258.

13. Al-leban W, Ali A, Shweliya H (2015): Epidemiological Study of Chronic Myeloid Leukemia Patients. IOSR J Pharm Biologi Sci., 10 (2): 2319-7676.

14. Willman C, Kang H, Potter J et al. (2005): A gene expression classifier for improved risk classification and outcome prediction in pediatric acute lymphblastic leukemia (ALL). Am Soci Hematol., 115: 225-230.

15. Ten Hacken E, Burger J (2016): Microenvironment interactions and B-cell receptor signaling in Chronic Lymphocytic Leukemia: Implications for disease pathogenesis and treatment. Biochimica et Biophysica 
Acta (BBA)-Molecular Cell Research, 1863 (3): 401413.

16. Prieto D, Oppezzo P (2017): Lipoprotein lipase expression in chronic lymphocytic leukemia: New insights into leukemic progression. Molecules, 22 (12): 2083-88.

17. Ahmed M, Hassab H (2008): Study of soluble CD44 and its expression by mononuclear cells in children with acute lymphoblastic leukemia: its relation to prognostic factors. Egypt J Immunol., 15 (2): 101-111.

18. Rezaieg N, Musleh M (2019): Assessment of the Role of Oxidative Stress and Circulating Biochemical markers in Childhood Leukemia. In Journal of Physics: Conference Series, 1294 (6): 62089-93.

19. Postovsky S, Arush M, Diamond E et al. (2003): The prevalence of low selenium levels in newly diagnosed pediatric cancer patients. Pediatr Hematol Oncol., 20: 273-80.

20. Ozgen I, Dagdemir A, Elli M et al. (2007): Hair selenium status in children with leukemia and lymphoma. Pediatr Hematol Oncol., 29 (8): 519-522.

21. Singh V, Kharb S, Ghalaut $P$ et al. (2005): Serum vitamin $\mathrm{E}$ in chronic myeloid leukaemia. Pediatr Blood Cancer, 44: 378-85.

22. Demir C, Demir H, Esen R et al. (2011): Altered serum levels of elements in acute leukemia cases in Turkey. Asian Pac J Cancer Prev., 12 (12), 3471-4.

23. Wysokinski D, Blasiak J, Wozniak K (2012): Zinc differentially modulates DNA damage induced by anthracyclines in normal and cancer cells. Experimental Oncology, 34 (4): 327-31.
24. Dayer D, Asadi Z, Samie M et al. (2015): Evaluation of serum copper levels in patients with leukemia and lymphoma. DAMA International, 4: 266- 70.

25. Akhgarjand C, Djafarian K, Rezvani H et al. (2018): Comparing serum levels of zinc, copper, certain antioxidant vitamins and dietary intakes in acute lymphoblastic leukemia (ALL) patients before and after chemotherapy. Am J Blood Res., 8 (3): 21-24.

26. Kennedy D, Ladas E, Rheingold $S$ et al. (2005): Antioxidant status decreases in children with acute lymphoblastic leukemia during the first six months of chemotherapy treatment. Pediatric Blood \& Cancer, 44 (4): 378-385.

27. Misaki K, Takitani K, Ogihara T et al. (2003): Alpha-tocopherol content and alpha-tocopherol transfer protein expression in leukocytes of children with acute leukemia. Free Radic Res., 37 (9):1037-42.

28. Sgarbieri U, Fisberg $M$, Tone $L$ et al. (2006): Nutritional assessment and serum zinc and copper concentration among children with acute lymphocytic leukemia: a longitudinal study. Sao Paulo Med J., 124: 316-20.

29. Prasad K, Cole W, Kumar B et al. (2001): Scientific Rationale for Using High-Dose Multiple Micronutrients as an Adjunct to Standard and Experimental Cancer Therapies. J Am Coll Nutr., 20 (5): 450-63.

30. Möricke A, Reiter A, Zimmermann M et al. (2008): Risk-adjusted therapy of acute lymphoblastic leukemia can decrease treatment burden and improve survival: treatment results of 2169 unselected pediatric and adolescent patients enrolled in the trial ALL-BFM 95. Blood J Am Soci Hematol., 111 (9): 4477-89. 